\title{
Síndrome de Tapia posterior a cirugía nasal
}

\section{Tapia syndrome post nasal surgery}

\author{
Rodrigo Cabezón A. ${ }^{1}$, Alejandra del Río R. ${ }^{2}$
}

\section{Resumen}

El síndrome de Tapia es una complicación poco frecuente secundaria a la manipulación de la vía aérea. Se caracteriza por haber una lesión concomitante de los pareas craneales X (nervio vago) y XII (nervio hipogloso), usualmente por compresión o sobredistensión de estos. Inicialmente puede hacernos sospechar una lesión central, al haber compromiso de dos nervios craneales bajos en forma simultánea, pero la gran mayoría de los casos descritos son lesiones periféricas. De los procedimientos asociados a esta complicación, los que lideran en frecuencia son los de cabeza y cuello, por lo que es de gran importancia tenerlo en conocimiento en el desarrollo de nuestra práctica clínica. Nuestro paciente presentó esta complicación tras una septoplastía con turbinectomía sin complicaciones en el sitio operatorio, ni anestésicas. Se manejó con fonoaudiología y corticoides orales, con recuperación completa a los cuatro meses de posoperatorio.

Palabras clave: síndrome de Tapia, parálisis cordal, parálisis hipogloso, septoplastía, disfonía.

\begin{abstract}
Tapia's Syndrome is a rare complication secondary to airway manipulation. It is characterized by a concurrent lesion of cranial nerve pairs X (vagus nerve) and XII (hypoglossal nerve), usually attributed to compression or stretching of these nerves. Initially, it may lead us to suspect a central lesion, as there is simultaneous involvement of two low cranial nerves, but the vast majority of cases described are peripheral lesions. The procedures most frequently associated with this complication are head and neck surgery, which is why it is very important to bear this in mind in the development of our clinical practice. Our patient showed Tapia's syndrome following septoplasty with turbinectomy without complications in the operative site nor under anesthesia. He was treated with phoniatric and oral corticoids, recovering completely four months after surgery.

Keywords: Tapia Syndrome, hypoglossal nerve paralysis, vocal cord paralysis, septoplasty, dysphonia.
\end{abstract}

'Departamento de Otorrinolaringología, Clínica Las Condes. Santiago, Chile. ${ }^{2}$ Facultad de Medicina, Universidad del Desarrollo. Santiago, Chile.

Los autores declaran no tener conflictos de interés.

Recibido el 3 de octubre de 2019. Aceptado el 24 de abril de 2020.

Correspondencia: Rodrigo Cabezón A. Lo Fontecilla 441, Las Condes. Santiago, Chile. Email: racabezo@gmail.com

\section{Introducción}

El síndrome de Tapia fue descrito por primera vez en 1904 por Antonio García Tapia, otorrinolaringólogo español, en un paciente posterior a un accidente con el cuerno de un toro a nivel cervical ${ }^{1}$. Se describe el síndrome de Tapia central que ocurre por compromiso del núcleo ambiguo ${ }^{2,3}$ y el periférico, que ocurre tras una lesión usualmente por compresión o sobredistensión de los nervios laríngeo recurrente, rama del nervio vago (X) e hipogloso $(\mathrm{XII})^{4}$. Tras la lesión se produce una paresia o parálisis a nivel lingual y laríngeo, usual- mente unilateral y habitualmente precedida por intubación orotraqueal ${ }^{5}$. Los síntomas más característicos son disfonía y disfagia ${ }^{6}$. Al examen físico destaca desviación lingual en protrusión, ipsilateral a la lesión, disminución de la motilidad lingual y en lesiones crónicas hasta se podría apreciar atrofia de la hemilengua comprometida. En la nasofibroscopía, se observa parálisis de la cuerda vocal ipsilateral a la lesión lingual. Al ser secundario a manipulación de la vía aérea, podría ocurrir en cualquier cirugía o soporte ventilatorio ${ }^{2}$, pero se observa más frecuentemente en cirugías de cabeza y cuello, de columna vertebral y tórax ${ }^{3,6}$. 


\section{Caso Clínico}

Paciente de sexo masculino, 26 años, sin antecedentes mórbidos, que consulta al otorrinolaringólogo por obstrucción nasal izquierda persistente. Presenta examen físico, tomografía computada (TC) de cavidades paranasales y rinomanometría compatibles con desviación septal izquierda severa e hipertrofia de cornetes, por lo que se indica septoplastía y turbinectomía. La cirugía se realiza en julio de 2018, y se lleva a cabo sin inconvenientes del punto de vista quirúrgico, ni de anestesia. La evolución del sitio quirúrgico posintervención fue favorable, sin embargo, en el posoperatorio inmediato se evidencia notoria disfonía y disfagia, al examen físico destaca desviación lingual a izquierda en protrusión (Figura 1).

Se realiza nasofibroscopía para evaluar la disfonía, evidenciando una parálisis cordal izquierda con compensación contralateral insuficiente y hiato fonatorio. Por compromiso de dos pares craneanos bajos, se solicita evaluación por neurología y se realiza una resonancia magnética de cerebro con énfasis en fosa posterior y de cuello con protocolo de nervio laríngeo recurrente, descartando lesiones de origen central o tumorales, por lo que se diagnostica un síndrome de Tapia izquierdo de origen periférico. Con esta hipó-

Figura 1. Se aprecia desviación lingual a izquierda, compatible con parálisis del XII nervio craneal. tesis diagnóstica en mente, se inician de forma empírica corticoides orales (prednisona $1 \mathrm{mg} /$ $\mathrm{kg} /$ día por una semana) y se envía al paciente a terapia fonoaudiológica para rehabilitación vocal y de motilidad lingual.

Paciente logra compensación fonatoria a las dos semanas postquirúrgicas, aunque con persistencia de parálisis cordal. También se aprecia una rápida recuperación de la movilidad lingual. En control con nasofibroscopía a los cuatro meses se observa recuperación completa de la movilidad cordal.

\section{Discusión}

El síndrome de Tapia es una complicación secundaria a la manipulación de la vía aérea, poco frecuente ${ }^{7}$. Ocurre tras la compresión o sobredistensión de los nervios craneales X y XII. Ha sido descrita tras packing faríngeo, sobredistensión del cuff del tubo orotraqueal o por apoyo de la curvatura del tubo orotraqueal, agudizado tras flexión de la cabeza del paciente durante la cirugía ${ }^{7}$. El sitio anatómico donde ocurre esta lesión es sobre la pared lateral de la base de la lengua y la hipofaringe ${ }^{3}$, específicamente en el cruce de ambos nervios, a nivel del ganglio nodoso del nervio vago, cercano al proceso transverso de $1^{\circ}$ vértebra cervical. Existe la descripción de varios casos de este síndrome en la literatura (Tabla 1).

Se realizó una revisión de los casos publicados en PubMed y LILACS, utilizando como palabra de búsqueda "Tapia syndrome" y "síndrome de tapia" respectivamente. De las publicaciones encontradas, posteriormente, se buscó de forma dirigida los casos citados por éstas, según su título de la publicación. En nuestra revisión obtuvimos 37 casos, de los cuales la mayoría se relacionan a intubación orotraqueal, por procedimientos quirúrgicos o soporte ventilatorio en UCI. De los procedimientos que más se asocian a esta complicación se encuentran en primer lugar, cabeza y cuello $(35,1 \%)$, seguido por procedimientos de columna $(24,3 \%)$ y cirugías de tórax $(18,9 \%)$. De los pacientes afectados, 29 son hombres $(78,4 \%)$ y 8 mujeres. La edad promedio fue de 43,7 años con un mínimo de 15 años y un máximo de 95 . El 48,7\% fueron lesiones izquierdas, $35,1 \%$ derechas y $13,5 \%$ 


\section{Tabla 1. Caracterización de casos publicados de síndrome de Tapia}

\begin{tabular}{|c|c|c|c|c|c|c|}
\hline Autor & Sexo & Edad & Procedimiento & Compromiso & Duración & Recuperación \\
\hline Kyung-Jee y cols. ${ }^{8}$ & Masculino & 64 & Laminoplastía & Izquierdo & 3 meses & $S / D$ \\
\hline M. Coninckx y cols. ${ }^{3}$ & Masculino & 64 & Neumonía y distrés respiratorio & Izquierdo & 22 meses & Completa \\
\hline M. Coninckx y cols. ${ }^{3}$ & Masculino & 49 & Fibrilación ventricular & Bilateral & 4 meses & Completa \\
\hline Turan y cols. ${ }^{9}$ & Masculino & 15 & Neumonía y distres respiratorio & $S / D$ & $S / D$ & $S / D$ \\
\hline Mayer y cols. ${ }^{10}$ & Masculino & 51 & Hernia hiatal y neumonía & Derecho & $S / D$ & $S / D$ \\
\hline Gelmers y cols. ${ }^{11}$ & Masculino & 41 & Bypass coronario & Izquierdo & S/D & $S / D$ \\
\hline Gelmers y cols. ${ }^{11}$ & Masculino & 36 & Toracotomía por bronquectasias & Izquierdo & 1 semana & Incompleta \\
\hline Boisseau y cols. ${ }^{12}$ & Masculino & 42 & Artroscopía de hombro & Izquierdo & 6 meses & Completa \\
\hline Yavuzer y cols. ${ }^{13}$ & Femenino & 42 & Rinoplastía & Izquierdo & 6 meses & Incompleta \\
\hline Cinar y cols. ${ }^{14}$ & Masculino & 20 & Rinoplastía & Bilateral & 1 mes & Completa \\
\hline Tesei y cols. ${ }^{15}$ & Femenino & 30 & Rinoplastía & Derecho & 4 meses & Completa \\
\hline Sotiriou y cols. ${ }^{16}$ & Femenino & 52 & Bypass coronario & Bilateral & 5 meses & Completa \\
\hline Rotondo y cols. ${ }^{17}$ & Masculino & 72 & Reemplazo de válvula aórtica & Izquierdo & 3 meses & Incompleta \\
\hline Kashyap y cols. ${ }^{18}$ & Masculino & 41 & Cirugía mandibular & Izquierdo & 16 meses & Incompleta \\
\hline Boga y cols. ${ }^{19}$ & Masculino & 35 & Rinoseptoplastía & Izquierdo & 15 días & Completa \\
\hline Park y cols. ${ }^{20}$ & Masculino & 42 & Columna cervical & Derecho & 7 meses & Incompleta \\
\hline Nalladaru y cols. ${ }^{21}$ & Masculino & 49 & Bypass coronario & Derecho & $\begin{array}{c}10 \\
\text { semanas }\end{array}$ & Completa \\
\hline Lykoudis and Seretis ${ }^{22}$ & Masculino & 32 & Rinoplastía & Derecho & 4 meses & Completa \\
\hline Lim y cols. ${ }^{23}$ & Masculino & 64 & Columna cervical & Izquierdo & 3 meses & Completa \\
\hline Gevogyan y Nedzelski7 & Femenino & 48 & Liposucción & Derecho & $S / D$ & $S / D$ \\
\hline Varedi y cols. ${ }^{24}$ & Masculino & 27 & Fractura zigomática & Izquierdo & 9 meses & Completa \\
\hline Park y cols. ${ }^{25}$ & Masculino & 53 & Columna cervical & Izquierdo & 6 meses & Completa \\
\hline Park y cols. ${ }^{25}$ & Masculino & 56 & Columna cervical & Izquierdo & 2 meses & Completa \\
\hline Ghorbani y cols. ${ }^{26}$ & Masculino & 27 & Rino-septoplastía & Izquierdo & 6 meses & Completa \\
\hline Mumtaz y cols. ${ }^{5}$ & Masculino & 23 & Extracción $3^{\text {er molar }}$ & Derecho & 3 meses & Completa \\
\hline Kang y cols. ${ }^{4}$ & Masculino & 47 & Columna cervical & Izquierdo & 8 meses & Incompleta \\
\hline Silva y cols. ${ }^{27}$ & Femenino & 41 & Columna cervical & Izquierdo & 1 año & Completa \\
\hline $\begin{array}{l}\text { Torres-Morientes y } \\
\text { cols. }{ }^{28}\end{array}$ & Masculino & 32 & Toracotomía & Derecho & 4 meses & Completa \\
\hline Al-Sihan y cols. ${ }^{29}$ & Masculino & 63 & $\begin{array}{c}\text { Estrechez de la arteria vertebral } \\
\text { derecha }\end{array}$ & Derecho & $S / D$ & Incompleta \\
\hline Johnson and Moore $\mathrm{e}^{30}$ & Masculino & 44 & Cirugía de hombro & Izquierdo & 6 meses & Completa \\
\hline McCleary ${ }^{31}$ & Femenino & 95 & Fractura de proceso odontoide & Bilateral & 1 año & Incompleta \\
\hline de Freitas y cols. ${ }^{32}$ & Femenino & 37 & $\begin{array}{l}\text { Paracoccidioidomicosis de } \\
\text { mucosa nasal izquierda }\end{array}$ & Izquierdo & 2 años & No \\
\hline Takimoto y cols. ${ }^{33}$ & Femenino & 18 & Radiación a tumor de cuello & Bilateral & 4 años & No \\
\hline Andrioli y cols..$^{34}$ & Masculino & 25 & $\begin{array}{l}\text { Neurofibroma bajo ganglio } \\
\text { nodoso }\end{array}$ & Derecho & $S / D$ & $S / D$ \\
\hline Cariati y cols. $^{2}$ & Masculino & 36 & Drenaje de absceso cervical & Derecho & $S / D$ & $S / D$ \\
\hline Cariati y cols. $^{2}$ & Masculino & 61 & Drenaje de absceso cervical & Derecho & $S / D$ & $S / D$ \\
\hline Cariati y cols. $^{2}$ & Masculino & 42 & $\begin{array}{c}\text { Reducción de fractura de } \\
\text { hombro }\end{array}$ & Derecho & $S / D$ & $S / D$ \\
\hline
\end{tabular}


bilaterales, uno sin información. Del total de casos, el $49 \%$ se recupera completamente, con un tiempo promedio de recuperación de 5,6 meses (15 días a 22 meses). El 21\% logra una recuperación incompleta y se describen 2 casos sin recuperación.

Nuestro caso en particular tiene las características promedio, tal como, sexo masculino, posterior a una cirugía nasal y con recuperación completa a los cuatro meses. No logramos identificar alguna de las posibles causas descritas, en particular intubación difícil o movilización excesiva de la cabeza. De todos modos, nos parece importante reportar el caso, ya que no existen casos reportados en Chile y, por lo infrecuente de la presentación, muchas veces se piensa como primera posibilidad en un evento de origen central, por lo cual es tranquilizador para el cirujano conocer que la gran mayoría de los casos son periféricos y tienen una evolución favorable. Cabe destacar la importancia de la rehabilitación fonoaudiológica en estos casos, para así lograr una recuperación más precoz y completa. En cuanto a la indicación farmacológica, en la mayoría de los casos se reporta uso concomitante de corticoides y/o vitamina $B$, sin embargo, por lo infrecuente de esta patología, no existe evidencia que respalde esta indicación, aunque fisiopatológicamente parece razonable.

\section{Conclusión}

El síndrome de Tapia es infrecuente, pero debemos tenerlo en cuenta en nuestra práctica quirúrgica. Si bien podría suceder después de cualquier procedimiento quirúrgico, se observa con mayor frecuencia en cirugías de cabeza y cuello. Es una complicación que en general tiene una recuperación favorable, donde la rehabilitación fonoaudiológica cumple un rol relevante.

\section{Bibliografía}

1. Schoenberg BS, Massey EW. Tapia's syndrome. The erratic evolution of an eponym. Arch Neurol. 1979;36(5):257-260. doi:10.1001/ archneur.1979.00500410035003.

2. Cariati P, Cabello A, Gálvez PP, Sánchez López D, García Medina B. Tapia’s syndrome: pathogenetic mechanisms, diagnostic management, and proper treatment: a case series. J Med Case Rep. 2016;10:23. doi:10.1186/s13256-016-0802-1.

3. Coninckx M, Cardoen S, Hemelsoet D. Tapia's syndrome in the intensive care unit: a rare cause of combined cranial nerve palsy following intubation. Acta Neurol Belg. 2015;115(4):533-537. doi:10.1007/ s13760-015-0500-6.

4. Kang JH, Kim DM, Kim SW. Tapia syndrome after cervical spine surgery. Korean J Spine. 2013;10(4):249251. doi:10.14245/kjs.2013.10.4.249.

5. Mumtaz S, Henry A, Singh M. Tapia’s Syndrome. Anesthesia Progress 2018;65(2):129-130.

6. Torres-Morientes L, Benito-Orejas J, Landinez G, Morais D. Síndrome de Tapia secundario a Toracotomía. Revista de la Sociedad Otorrinolaringológica de Castilla y León, Cantabria y La Rioja. Rev Soc Otorrinolaringol Castilla Leon Cantab La Rioja 2012; 3:48-59.

7. Gevorgyan A, Nedzelski JM. A late recognition of tapia syndrome: a case report and literature review. Laryngoscope. 2013;123(10):2423-2427. doi:10.1002/ lary.24070.

8. Kyung-Jee L, Kim M, Kang M, Lee H, Park E, Kwon $\mathrm{K}$, Lee S, Moon H. Tapia's syndrome following cervical laminoplasty: A case report. Korean J Anesthesiol. 2013;64(2):172-174.

9. Turan I, Yildirim ZK, Tan H. Bilateral Tapia syndrome secondary to oropharyngeal intubation. J Neurosurg Anesthesiol. 2012;24(1):78. doi:10.1097/ ANA.0b013e31823769ef.

10. Mayer A, Opran H. Letter: Tapia syndrome. JAMA. 1974;227(3):326. doi:10.1001/ jama.1974.03230160054024.

11. Gelmers HJ. Tapia's syndrome after thoracotomy. Arch Otolaryngol. 1983;109(9):622-623. doi:10.1001/archotol.1983.00800230058014.

12. Boisseau N, Rabaijaona H, Grimaud D, Raucoules-Aimé M. Tapia's syndrome following shoulder surgery. British Journal of Anaesthesia. 2002;88(6):869-70.

13. Yavuzer R, Basterzi Y, Ozkose Z, Demir Y, Yilmaz M, Ceylan A. Tapia's syndrome following septorhinoplasty. Aest Plast Surg. 2004; 28: 208-211.

14. Cinar SO, Seven H, Cinar U, Turgut S. Isolated bilateral paralysis of the hypoglossal and recurrent laryngeal nerves (Bilateral Tapia's syndrome) after transoral intubation for general anesthesia. Acta Anaesthesiol Scand. 2005;49(1):98-99. doi:10.1111/ j.1399-6576.2004.00553.x.

15. Tesei F, Poveda LM, Strali W, Tosi L, Magnani G, Farneti G. Unilateral laryngeal and hypoglossal paralysis (Tapia's syndrome) following rhinoplasty in general anaesthesia: case report and review of the literature. Acta Otorhinolaryngol Ital. 2006;26(4):219221.

16. Sotiriou K, Balanika M, Anagnostopoulou S, 
Gomatos C, Karakitsos D, Saranteas T. Postoperative airway obstruction due to Tapia's syndrome after coronary bypass grafting surgery. Eur J Anaesthesiol. 2006;24:378-379.

17. Rotondo F, De Paulis S, Modoni A, Schiavello R. Peripheral Tapia's syndrome after cardiac surgery. Eur J Anaesthesiol. 2010;27:575-583.

18. Kashyap SA, Patterson AR, Loukota RA, Kelly G. Tapia's syndrome after repair of a fractured mandible. Br J Oral Maxillofac Surg. 2010;48(1):5354. doi:10.1016/j.bjoms.2009.01.021

19. Boğa I, Aktas S. Treatment, classification, and review of Tapia syndrome. J Craniofac Surg. 2010;21(1):278280. doi:10.1097/SCS.0b013e3181c678f0.

20. Park J, Ahn R, Weon YC, Yang Ds. Diagnosing Tapia Syndrome using a videofluoroscopic swallowing study and electromyography after anterior cervical spine surgery. Am J Phys Rehabil. 2011;90:948-953.

21. Nalladaru Z, Wessels A, DuPreez L. Tapia's syndrome: A rare complication following cardiac surgery. Interactive CardioVascular and Thoracic Surgery. 2012;14:131-132.

22. Lykoudis E, Seretis K. Tapia's syndrome: An unexpected but real complication of rhinoplasty: Case report and literature review. Aesth Plast Sur. 2012;36:557-559.

23. Lim K, Kim M, Kang M, Lee H, et al. Tapia’s syndrome following cervical laminoplasty. Korean J Anesthesiol. 2013;64:172-174.

24. Varedi P, Shirani G, Karimi A, Varedi P, Khiabani K, Bohluli B. Tapia syndrome after repairing a fractured zygomatic complex: a case report and review of the literature. J Oral Maxillofac Surg. 2013;71(10):16651669. doi:10.1016/j.joms.2013.05.019.

25. Park CK, Lee DC, Park CJ, Hwang JH. Tapia's Syndrome after Posterior Cervical Spine Surgery under General Anesthesia. J Korean Neurosurg Soc. 2013;54(5):423-425. doi:10.3340/jkns.2013.54.5.423.

26. Ghorbani J, Dabir S, Givehchi G, Najafi M. Co- presentation of Tapia's syndrome and pressure alopecia. A rare event after septorrhinoplasty: A case report and literature review. Acta Anaesthesiologica Taiwanica. 2014;52:38-40.

27. Silva AH, Bishop M, Krovvidi H, Costello D, Dhir J. Tapia syndrome: an unusual complication following posterior cervical spine surgery. Br J Neurosurg. 2019;33(2):217-218. doi:10.1080/02688697.2017.1318 109.

28. Torres-Morientes L, Benito-Orejas J, Landinez G, Morais D. Síndrome de Tapia secundario a toracotomía. Revista de la Sociedad Otorrinolaringológica de Castilla y León, Cantabria y La Rioja. 2011;2.

29. Al-Sihan M Jr, Schumacher M, Löhle E. Tapia syndrome caused by a vertebral artery dissection. Ear Nose Throat J. 2011;90(7):313-314. doi:10.1177/014556131109000709.

30. Johnson TM, Moore HJ. Cranial nerve X and XII paralysis (Tapia's syndrome) after an interscalene brachial plexus block for a left shoulder Mumford procedure. Anesthesiology. 1999;90(1):311-312. doi:10.1097/00000542-199901000-00040.

31. Mc Cleary A. A fracture of the odontoid process complicated by tenth and twelfth cranial nerve palsies. Spine. 1993;18(7):932-935.

32. de Freitas MR, Nascimento OJ, Chimelli L. Tapia's syndrome caused by Paracoccidioidis brasiliensis. $J$ Neurol Sci. 1991;103(2):179-181. doi:10.1016/0022510x(91)90161-y.

33. Takimoto T, Saito Y, Suzuki M, Nishimura T. Radiation-induced cranial nerve palsy: hypoglossal nerve and vocal cord palsies. J Laryngol Otol. 1991;105(1):44-45. doi:10.1017/s0022215100114793.

34. Andrioli G, Rigobello L, Mingrino S, Toso V. Tapia's syndrome caused by a neurofibroma of the hypoglossal and vagus nerves: case report. J Neurosurg. 1980;52(5):730-732. doi:10.3171/ jns.1980.52.5.0730. 